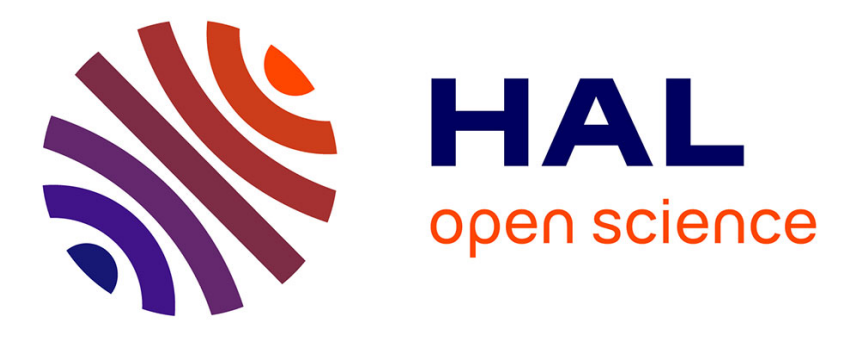

\title{
Removing ring artefacts from synchrotron radiation-based hard x-ray tomography data
}

Christos Bikis, Georg Schulz, Pierre Paleo, Alessandro Mirone, Alexander

Rack, Bert Müller, Peter Thalmann, Stefan Siegrist, Emre Cörek, Jörg Huwyler

\section{To cite this version:}

Christos Bikis, Georg Schulz, Pierre Paleo, Alessandro Mirone, Alexander Rack, et al.. Removing ring artefacts from synchrotron radiation-based hard x-ray tomography data. SPIE Conference on Optics and Photonics, Aug 2017, San Diego, United States. pp.1039114, 10.1117/12.2274236 hal-01815229

\section{HAL Id: hal-01815229 \\ https://hal.science/hal-01815229}

Submitted on 18 Jul 2018

HAL is a multi-disciplinary open access archive for the deposit and dissemination of scientific research documents, whether they are published or not. The documents may come from teaching and research institutions in France or abroad, or from public or private research centers.
L'archive ouverte pluridisciplinaire HAL, est destinée au dépôt et à la diffusion de documents scientifiques de niveau recherche, publiés ou non, émanant des établissements d'enseignement et de recherche français ou étrangers, des laboratoires publics ou privés. 


\title{
Removing ring artefacts from synchrotron radiation-based hard X-ray tomography data
}

\author{
Peter Thalmann ${ }^{a}$, Christos Bikis $^{a}$, Georg Schulz $^{a}$, Pierre Paleo $^{b}$, Alessandro Mirone $^{b}$, \\ Alexander Rack ${ }^{b}$, Stefan Siegrist ${ }^{c}$, Emre Cörek ${ }^{c}$, Jörg Huwyler ${ }^{c}$, and Bert Müller \\ ${ }^{a}$ Biomaterials Science Center, University of Basel, Allschwil, Switzerland; \\ ${ }^{b}$ European Synchrotron Radiation Facility, Grenoble, France \\ ${ }^{c}$ Department of Pharmaceutical Sciences, University of Basel, Basel, Switzerland
}

\begin{abstract}
In hard X-ray microtomography, ring artefacts regularly originate from incorrectly functioning pixel elements on the detector or from particles and scratches on the scintillator. We show that due to the high sensitivity of contemporary beamline setups further causes inducing inhomogeneities in the impinging wavefronts have to be considered. We propose in this study a method to correct the thereby induced failure of simple flatfield approaches. The main steps of the pipeline are (i) registration of the reference images with the radiographs (projections), (ii) integration of the flat-field corrected projection over the acquisition angle, (iii) high-pass filtering of the integrated projection, (iv) subtraction of filtered data from the flat-field corrected projections. The performance of the protocol is tested on data sets acquired at the beamline ID19 at ESRF using single distance phase tomography.
\end{abstract}

Keywords: ring artefacts, tomography, 2D-2D registration, in-line tomography, high-pass filter, phase reconstruction

\section{INTRODUCTION}

In hard X-ray microtomography, ring artefacts are a common unwanted phenomenon that can severely decrease the obtained image quality. Frequently, they originate from incorrectly functioning pixel elements on the detector unit or from particles and scratches on the scintillator. The high level of sensitivity achieved by nowadays beamline setups does not only allow to be more sensitive to density variations within the specimen, but also turns the imaging setups more susceptible to inhomogeneities introduced on the impinging wavefronts. In detail, inadequacies of the X-ray optical elements like windows, attenuators and distortions of the wavefront by the sample itself can cause a partial failure of simple flatfield correction. Thus far, many ring artefacts removal algorithms are applied on the sinogram ${ }^{1}$ or the reconstruction slice ${ }^{2}$ itself. A better approach would be however to not only to remove the artefacts themselves, but also to maintain the radiographs true information.

All specimens investigated were composed of low-absorbing tissues, such that typical hard X-ray micro computed tomography in absorption contrast mode does not provide sufficient contrast to adequately resolve the inner structure of the specimen investigated. However, light propagation through a medium can be described by the complex refractive index

$$
n(x, y, z)=1-\delta(x, y, z)+i \beta(x, y, z),
$$

where the imaginary part, $\beta$, accounts for the attenuation and the refractive index decrement, $\delta$, accounts for the phase shift of the impinging wave respectively. ${ }^{3}$ It is well known, that for materials composed of low-Z elements, as is the case for the specimens investigated, the total phase-shift cross section can be up to three orders of magnitude higher then the total absorption cross section depending on the photon energy ${ }^{4,5}$ making phase tomography the preferential method for the tree-dimensional investigation of such specimens. There are several techniques for the phase retrieval, as for instance grating interferometry ${ }_{6}{ }^{6,7}$ in-line single distance and holotomograhy. ${ }^{8,9}$

Further author information: (Send correspondence to Bert Müller)

Bert Müller: E-mail: bert.mueller@unibas.ch, Telephone: 0041612075430 
The motivation behind developing such a ring-artefact removal approach lies on the fact that single-distance phase retrieval, i.e. our X-ray tomography modality of choice for the three-dimensional investigation of physically soft tissues, including nervous tissue, is more prone to such artefacts than its alternatives (e.g. grating interferometry). Nevertheless, the comparative advantages offered, as will be described in what follows, compensate for this specific shortcoming, which we now prove that can be tackled in an efficient manner.

The reason for single distance in-line, propagation-based tomography being the method of choice lies in the superior spatial resolution provided, simpler instrumentation and very efficient scanning times that are absolutely necessary for biomedical applications requiring several replicates to be scanned for a specific experiment. In in-line propagation-based phase tomography the recorded projections contain both absorption and phase information, where the phase information can be interpreted as the Laplacian of the phase of the wavefront after transversing the sample. In 2002, Paganin et al. ${ }^{10}$ derived an algorithm based on the transport of intensity equation (TIE) ${ }^{11}$ assuming a single-component specimen and that the object and the detector plane fulfill the near-field condition. and requiring only one single distance. Despite the violation of the single-component assumption, the algorithm has proven itself to be robust also for the case of biological specimens, and soft tissue in particular. ${ }^{8,9}$ It also presents the advantage of requiring only one measurement at a single distance.

Here, we thus introduce an improved ring artefact removal algorithm for high-resolution micro computed tomography. The performance of the algorithm is illustrated using synchrotron radiation-based micro computed tomography $(\mathrm{SR} \mu \mathrm{CT})$ in phase contrast mode.

\section{MATERIALS AND METHODS}

\subsection{Specimen preparation}

\subsubsection{Mouse brain tumor}

For the experiment a nude mouse (CD-1-Foxn1nu, Charles River Laboratories, France) was used. A stereoactic frame for head fixation was used for the injection of murine glioma cells (2104 GL261 cells) into the right frontal lobe. In-vivo magnetic resonance experiments were performed using a PharmaScan 47/16 MRI system equipped with a $4.7 \mathrm{~T}$ magnet with $16 \mathrm{~cm}$ bore diameter and a cryogenic quadrature RF surface transmit/receive coil (Bruker BioSpin Ettlingen, Germany). During in-vivo experiments, the mouse was placed on a customized support equipped with a stereotactic system for head fixation and a face mask for anaesthetic administration (isofluorane (1.5\% to $2 \%$ ). For the administration of contrast agent a cannula was inserted into the right tail vein. All the experiments were approved by local authorities (license ZH 168/2010) and performed in strict adherence to the Swiss law for animal protection. The mouse was euthanized by cervical dislocation at day 15 following tumor inoculation. A specimen containing brain and tumor was explanted using augmented reality assistance $^{12}$ and was fixed in buffered formalin immediately after extraction. For the tomography measurements, a dedicated plastic container was used.

\subsubsection{Human nerve}

A human peripheral nerve was obtained post-mortem from a donated body, with informed consent for scientific use provided beforehand. All procedures were conducted in accordance with the Declaration of Helsinki and approved by the Ethikkommission Nordwestschweiz. Following standard pathology procedure, the peripheral nerve was extracted from the donated body and fixed in $4 \%$ histological-grade buffered formalin. It was subsequently dehydrated and finally embedded in a paraffin/plastic polymer mixture. For the tomography measurement, a cylindrical sample with a diameter of $6 \mathrm{~mm}$ was extracted from the paraffin block, using a metal punch.

\subsubsection{Zebra fish embryo}

Experiments were conducted using the zebrafish wild type strain AB/Tbingen (AB/TU). Adult zebrafish were kept in aerated tanks tempered to $28.0^{\circ} \mathrm{C}$ at a $10: 14$ hour light/dark cycle. After collection, eggs were cleaned, sorted and transferred into petri dishes with $0.5 \mathrm{X}$ embryo-medium (E2 medium, ${ }^{13} 5.0 \mathrm{mM} \mathrm{NaCl}, 0.25 \mathrm{mM} \mathrm{KCl}$, $0.5 \mathrm{mM} \mathrm{MgSO}_{4}, 0.15 \mathrm{mM} \mathrm{KH}_{2} \mathrm{PO}_{4}, 0.05 \mathrm{mM} \mathrm{Na}_{2} \mathrm{HPO}_{4}, 0.5 \mathrm{mM} \mathrm{CaCl}, 0.71 \mathrm{mM} \mathrm{NaHCO} 3,1.01$ water (Millipore 18-textohm), $0.001 \%(\mathrm{w} / \mathrm{v})$ methylene blue, $\mathrm{pH}$ 7.4). Petri dish containing eggs were stored in an incubator 
Table 1. Experimental and reconstruction parameters, where $\delta / \beta$ denotes the assumed ratio of refractive index decrement over the imaginary part of the refractive index for each specimen, $d$ the sample-detector distance, $N$ the total number of projections acquired, and $t$ the exposure time per projection

\begin{tabular}{|c|c|c|c|c|c|c|c|c|}
\hline Specimen & Pixel size $[\mu \mathrm{m}]$ & $\delta / \beta$ & $d[\mathrm{~mm}]$ & $N$ & $t[\mathrm{~s}]$ & off-axis & preparation & camera \\
\hline Brain tumor & 1.87 & 1546 & 202 & 2000 & 0.2 & no & formalin & FReLoN 2K \\
\hline Human nerve & 0.66 & 1500 & 30 & 5120 & 0.1 & yes & paraffin & PCO Edge \\
\hline Zebrafish & 0.33 & 2000 & 10 & 5120 & 0.1 & yes & paraffin & PCO Edge \\
\hline
\end{tabular}

(Aqualytic, Dortmund, Germany) at $28.0^{\circ} \mathrm{C}$. E2 medium was renewed daily. Zebrafish embryos were dechorionated $24 \mathrm{~h}$ post fertilization (hpf) by removal of the chorion with acupuncture needles $(0.20 \mathrm{~mm} \times 15 \mathrm{~mm}$, Wandrey, Berlin, Germany). The dechorionated embryos were immobilized 48 hpf by adding $1 \mathrm{ml} 25 \mathrm{X}$ tricaine stock solution to the medium (400.0 mg tricaine methanesulfonate, $97.9 \mathrm{ml}$ water (Millipore 18-textohm), $2.1 \mathrm{ml}$ trisaminomethane, $\mathrm{pH}=9$ ). Immobilized zebrafish embryos were fixed in $4.0 \%$ paraformaldehyde and dehydrated in ethanol. Fixed zebrafish embryos were aligned in the channel (diameter $0.8 \mathrm{~mm}$ ) of a two-pieced custom-made aluminum mold $(3.0 \mathrm{~cm} \times 2.0 \mathrm{~cm} \times 2.0 \mathrm{~cm})$. Aligned embryos were air dried for one minute to ensure complete evaporation of excess ethanol. Thereafter, the mold was placed vertically into liquid paraffin wax $\left(80.0^{\circ}\right.$, SigmaAldrich, Buchs, Switzerland) allowing the channel containing the zebrafish embryo to fill with paraffin due to capillary forces. After cooling, the newly formed paraffin rod containing the embedded embryo was removed from the socket of the mold with a blunt syringe. The paraffin rods were stored at $4.0^{\circ} \mathrm{C}$ until further use.

\subsection{Single-distance phase retrieval}

All specimens were measured using in-line single distance phase tomography with the experiments being carried out at the ID19 beamline (ESRF, Grenoble, France). Phase retrieval was performed using the algorithm proposed by Paganin et al. ${ }^{10}$ using ANKAphase. ${ }^{14} \mathrm{~A}$ list of the acquisition and reconstruction parameters is given in Table 1. The phase projections were reconstructed using the standard filtered back-projection algorithm ${ }^{15}$ implemented in MATLAB (2016a, The MathWorks, Inc., Natick, Massachusetts USA).

\section{RESULTS AND DISCUSSION}

Figure 1 shows selected phase reconstruction slices for for the three specimen, before ring artefact correction. The slices contain severe ring artifacts. As already stated, usually, these artefacts arise from incorrectly working scintillator or pixel elements. In such cases they can be removed by using a stripe removing filter on the
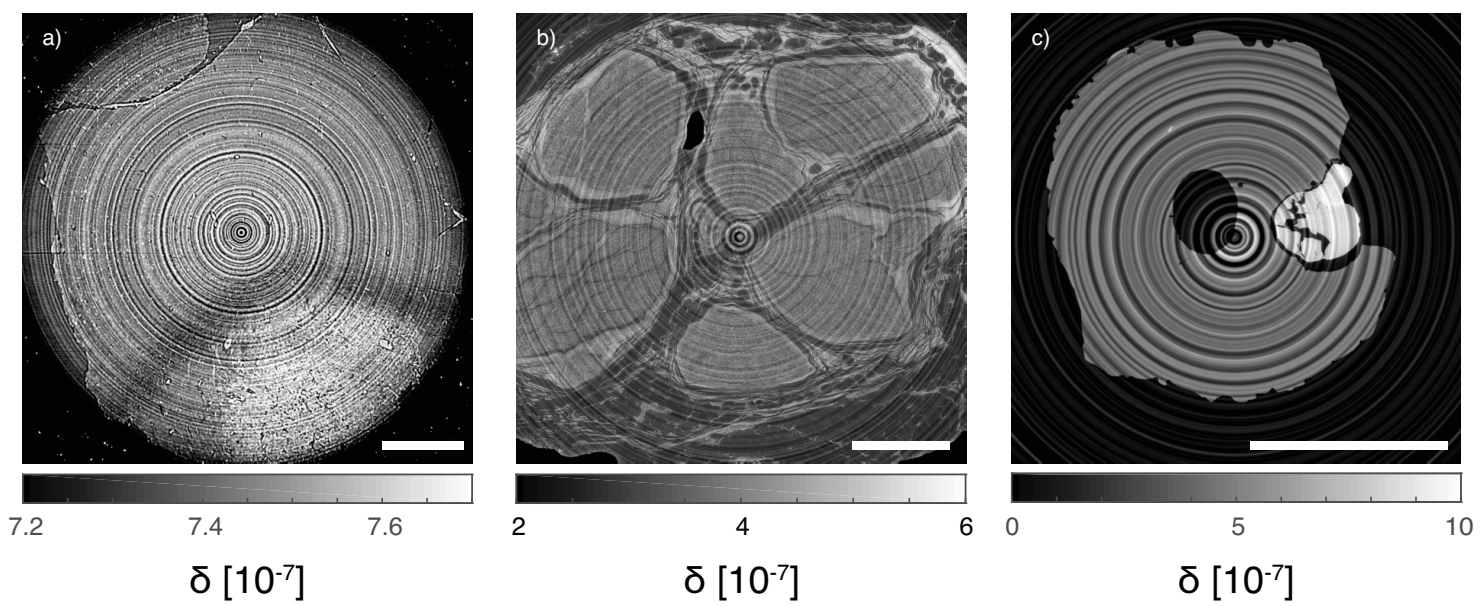

Figure 1. The images show characteristic slices of the phase-reconstructed specimens with standard flat-field correction applied: (a) brain tumor, (b) human nerve and (c) zebra fish embryo. All slices contain more or less strong ring artifacts. Scale bar length is $500 \mu \mathrm{m}$. 

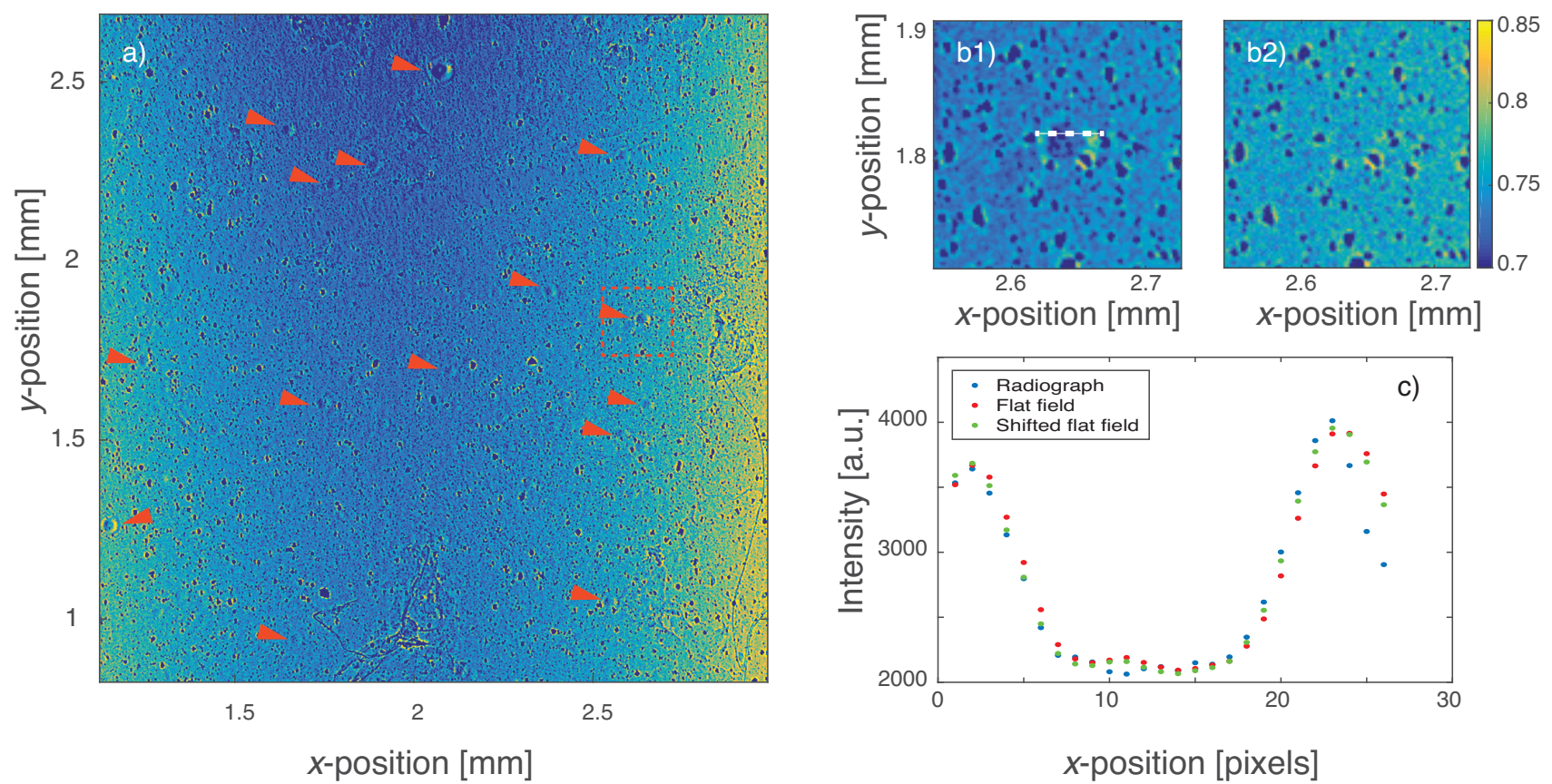

Figure 2. (a) The image shows a selected region of interest (ROI) of a representative projection of the mouse brain tumor specimen. The read arrowheads indicate the prominent donut-like artefacts that persist after standard flat-field correction. A zoom-in of the region inside the dashed box containing such a donut-like structure prior to and after having shifted the flat-field appropriately is given in (b1) and (b2) respectively. (c) A line plot through the donut-like artefact indicated by the red line in figure (b1) clearly shows the sub-pixel mismatch that occurs when simple flat-field correction is used.

sinogram ${ }^{1}$ or directly on the reconstruction slice. ${ }^{2}$ However, measuring low-absorbing specimens in phasecontrast mode requires high sensitivity, provided by nowadays beamline setups. The high sensitivity achieved makes the system also more prone to disturbances of the impinging wavefronts. Under these circumstances simple flat-field correction is insufficient.

To better elucidate why this happens, consider for example the projection of the mouse brain tumor, given in Figure 2(a). Numerous donut-like artefacts can be observed, that are clearly artificial. Having a closer look on the line plot through such a structure given in 2(b) reveals a sub-pixel mismatch of the flat-field (red) with its projection (blue), as the plots slightly deviate where the curve is falling and rising. Shifting the flat-field to its corresponding projection (green) resulted in almost precise match of the two images as can also be seen in Figure 2(b), while the shift in $x$-direction was around 0.2 pixels and in the $y$-direction less than 0.1 pixels.

The effect of using translation registration to correct for the mismatch is also illustrated in Figure 3. While for the mouse brain specimen measured with a pixel size of $1.87 \mu \mathrm{m}$ translation registration mainly resulted in the removal of several donut-like structures, its effect with decreasing pixel size was much more pronounced. For example, for the zebra fish embryo measurement, a translation over a pixel resulted in the removal of prominent cloud-like structures on the projection and thereby resulted in projection containing many more details. However, for all measurements, image registration alone could not remove all ring artefacts, as can be seen in the sinogram comparison Figure 3 (c) as well as the corresponding reconstructed slices in Figure 4(b).

It should also not be overlooked that image registration can readily increase reconstruction time. For instance, finding the best out of 100 flat-field images for 5120 radiographs would require 512000 registrations, which is an extremely computation-intensive task and can be immensely time-consuming for commercialy available 

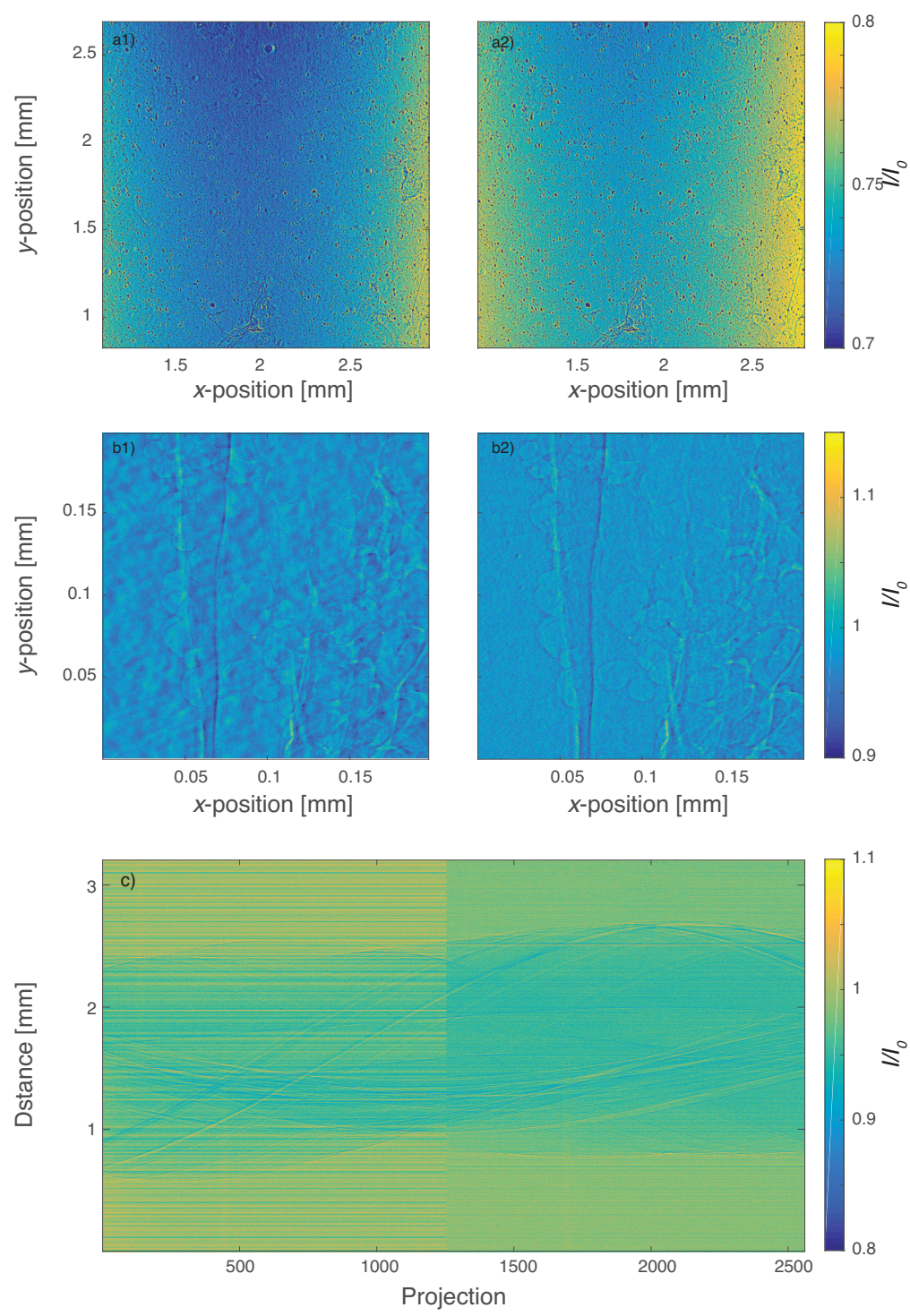

Figure 3. This figure illustrates the beneficial effect of flat-field registration on the projection quality. For the mouse brain tumour (a) most of the donut-like artifacts were removed and for the zebra fish embryo (b) flat-field registration was able to remove the dominant cloud-like artefacts, resulting in a much more detailed projection. A representative sinogram of the zebra fish embryo, given in (c), shows the improved efficiency of flat-field registration in removing all but the thinnest sinogram artefact lines. The first 1260 projections were produced using simple flat-field correction and the second 1260 projections were achieved using flat-field registration prior to the standard correction.

workstations. Therefore, we tested different registration approaches, in an effort to decrease computation time. The parameters investigated were the registration method itself, the metric, the optimizer as well as the number of registrations per radiograph. All registrations were performed using the Insight Segmentation and Registration Toolkit (ITK). ${ }^{16}$ In terms of method, neither the affine nor the similarity registration yielded better results than the Translation registration in terms of the obtained similarity. For instance, the final rotation after Similarity registration remained below $0.001^{\circ}$. In terms of speed and quality the Mattes mutual information in combination with the Powell optimizer was found to be the most suitable choice. Furthermore, taking the median over a flat-field block to register to any given projection resulted in an increase of the similarity value, compared to taking a random flat-field. Additionally, the weighting of nearest flat-field blocks can increase similarity, but the optimal weighting did not necessarily correlate with the theoretical one. With the suggested parameters 
one single registration took about $1 \mathrm{~s}$ on a workstation using six Intel(R) Xeon(R) CPU E5-2620 v3 @ 2.40GHz processors. Hence, assuming five registrations per projection to find the optimal weighting, the entire process takes about $8 \mathrm{~h}$ to $24 \mathrm{~h}$, depending on the projection contrast, for 5120 radiographs. So far non-rigid registration has not been tested. The same holds for finding the optimally acquired flat-field as the algorithm has not been yet adapted for a cluster.

As already mentioned, registration by itself was insufficient to completely remove the ring artifacts. Thus, in a second step, we applied a frequency filter approach proposed by Mirone et al. ${ }^{17}$ For every single projection the median over a user-defined number of nearest projections is calculated. Then a high frequency filter is applied to the median averaged projection. The resulting high frequency image is then finally subtracted from the registration-corrected projection.
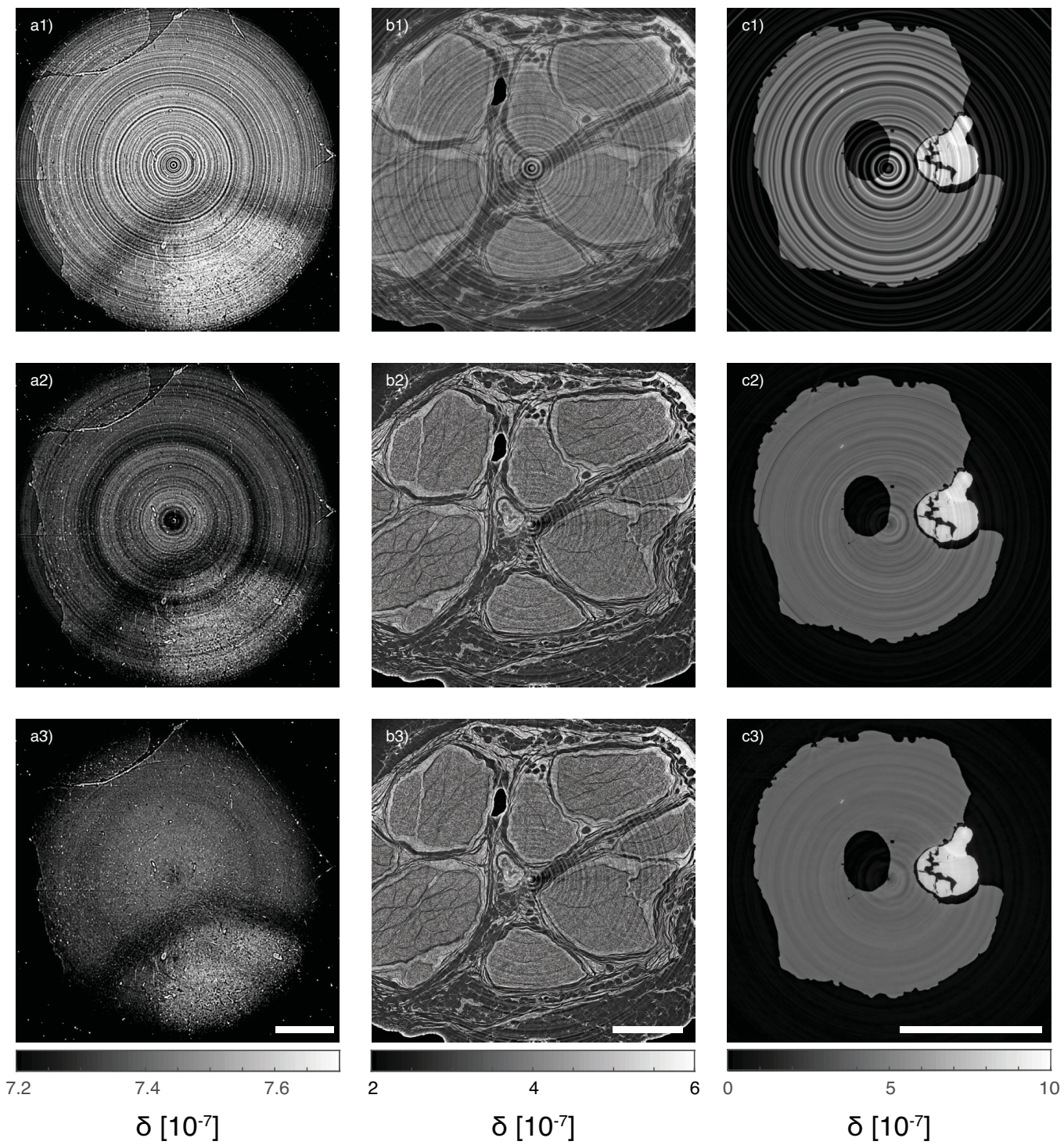

Figure 4. The effects of the ring artifact removal steps applied for (a) the mouse brain tumor, (b) the human nerve and (c) the zebra fish embryo, respectively. The top row shows representative slices for the specimens using simple flat-field correction. The middle row shows the same slices using translation registration prior to the flat-field correction and the bottom row shows the reconstructed slices obtained after the added step of high-pass filtering the flat-field corrected projections. Scale bar length is $500 \mu \mathrm{m}$. 
Our proposed algorithm exploits the complementarity between the registration process and the high frequency filter subtraction. In detail, the former is particularly efficient for removing thicker rings caused by bigger artefacts in the projection, while the later is especially effective for removing the remaining, thinner, ring artefacts.

\section{CONCLUSION}

We have implemented and tested a two-step procedure for ring artefact removal, based on the combination of flatfield translation registration and high-pass filtering of projections. This approach has been proven particularly effective for the visualization of several types of low absorbing samples, even using phase tomography modalities such as single distance phase retrieval, which are generally prone to ring artefacts. The implementation is both robust and time-efficient, allowing reconstruction of up to one dataset per day on a standard workstation. The main remaining challenge is optimizing the process to decrease computation time, as well as develop a userfriendly interface to allow for processing large series of datasets required for biomedical experiments with several specimen replicates.

\section{ACKNOWLEDGMENTS}

The authors thank Prof. Michele Bernasconi and Dr. Zsofia Kovacs from the University Children's Hospital in Zurich for providing the mouse brain tumor sample. The authors also thank Gabriel Schweighauser, Jürgen Hench and Stephan Frank from the Neuropathology Department of the Basel University Hospital for providing the human peripheral nerve sample and assisting in the special preparation required for the tomography measuremts. The authors also acknowledge the support of Dr. Irene Zanette from the Diamond Light Source during beamtime.

\section{FUNDING}

The financial contribution of the Swiss National Science Foundation (Project Nos. 144535 and 147172) and NanoReg2 (European Union Horizon 2020 research agreement 646221, 15.0200-3) is gratefully acknowledged as well as the provision of beamtime at beamline ID19 under experiment number MD-860 and MD-981 from the European Synchrotron Radiation Facility (ESRF).

\section{REFERENCES}

[1] Münch, B., Trtik, P., Marone, F., and Stampanoni, M., "Stripe and ring artifact removal with combined wavelet - fourier filtering," Opt. Express 17(10), 8567-8591 (2009).

[2] Sadi, F., Lee, S. Y., and Hasan, M. K., "Removal of ring artifacts in computed tomographic imaging using iterative center weighted median filter," Comput. Biol. Med. 40(1), 109-118 (2010).

[3] Attwood, D. T., [Soft X-Rays and Extreme Ultraviolet Radiation: Principles and Applications], Cambridge University Press (1999).

[4] Momose, A., "Phase-contrast X-ray imaging based on interferometry," J. Synchrotron Radiat. 9(3), 136-142 (2002).

[5] Als-Nielson, J. and McMorrow, D., [Elements of Modern X-ray Physics (2nd Edition)], John Wiley \& Sons Ltd. (2011).

[6] Weitkamp, T., Diaz, A., David, C., Pfeiffer, F., Stampanoni, M., Cloetens, P., and Ziegler, E., "X-ray phase imaging with a grating interferometer," Opt. Express 13(16), 6296-6304 (2005).

[7] Thalmann, P., Bikis, C., Hipp, A., Müller, B., Hieber, S. E., and Schulz, G., "Single and double gratingbased x-ray microtomography using synchrotron radiation," Appl. Phys. Lett. 110(6), 061103 (2017).

[8] Zanette, I., Lang, S., Rack, A., Dominietto, M., Langer, M., Pfeiffer, F., Weitkamp, T., and Müller, B., "Holotomography versus x-ray grating interferometry: A comparative study," Appl. Phys. Lett. 103(24), 244105 (2013).

[9] Lang, S., Zanette, I., Dominietto, M., Langer, M., Rack, A., Schulz, G., Le Duc, G., David, C., Mohr, J., Pfeiffer, F., Müller, B., and Weitkamp, T., "Experimental comparison of grating- and propagation-based hard x-ray phase tomography of soft tissue," J. Appl. Phys. 116(15), 154903 (2014). 
[10] Paganin, D., Mayo, S. C., Gureyev, T. E., Miller, P. R., and Wilkins, S. W., "Simultaneous phase and amplitude extraction from a single defocused image of a homogeneous object," J. Microsc. 206(1), 33-40 (2002).

[11] Teague, M. R., "Deterministic phase retrieval: a green's function solution," J. Opt. Soc. Am. 73(11), 1434-1441 (1983).

[12] Schneider, A., Thalmann, P., Pezold, S., Hieber, S. E., and Cattin, P. C., "Augmented reality assisted brain tumor extraction in mice," Lect. Notes Comput. Sci. , 255-264 (2015).

[13] Sieber, S., Siegrist, S., Schwarz, S., Porta, F., Schenk, S. H., and Huwyler, J., "Immobilization of enzymes on plga sub-micrometer particles by crosslinked layer-by-layer deposition," Macromol. Biosci. 17(8), 1700015 (2017).

[14] Weitkamp, T., Haas, D., Wegrzynek, D., and Rack, A., "ANKAphase: software for single-distance phase retrieval from inline X-ray phase-contrast radiographs," J. Synchrotron Radiat. 18(4), 617-629 (2011).

[15] Kak, A. C. and Slaney, M., [Principles of computerized tomographic imaging], IEEE Press (1988).

[16] ITK. (2016). The Insight Segmentation and Registration Toolkit (ITK). Available online at: http://www.itk.org.

[17] Mirone, A., Brun, E., Gouillart, E., Tafforeau, P., and Kieffer, J., "The pyhst2 hybrid distributed code for high speed tomographic reconstruction with iterative reconstruction and a priori knowledge capabilities," 1st International Conference on Tomography of Materials and Structures 324, 41-48 (2014). 